\title{
NONASSOCIATIVE CYCLIC EXTENSIONS OF FIELDS AND CENTRAL SIMPLE ALGEBRAS
}

\author{
C. BROWN AND S. PUMPLÜN
}

\begin{abstract}
We define nonassociative cyclic extensions of degree $m$ of both fields and central simple algebras over fields. If a suitable field contains a primitive $m$ th (resp., $q$ th) root of unity, we show that suitable nonassociative generalized cyclic division algebras yield nonassociative cyclic extensions of degree $m$ (resp., $q s$ ). Some of Amitsur's classical results on non-commutative associative cyclic extensions of both fields and central simple algebras are obtained as special cases.
\end{abstract}

\section{INTRODUCTION}

Analogously as both for commutative field extensions [6, 2, 3, 26] and for associative cyclic extensions of fields and central simple algebras [5], nonassocative cyclic extensions of degree $m$ of a field or a central division algebra are investigated separately for prime characteristics and for the case that the characteristic is zero or a prime $p$ with $\operatorname{gcd}(p, m)=1$. Nonassociative cyclic extensions of degree $p$ in characteristic $p$ were already studied in [18].

Let $D$ be a finite-dimensional central division algebra over a field $K$. An (associative) central division algebra $A$ over a field $F$ is called a non-commutative cyclic extension of degree $m$ of $D$ over $K$, if $\operatorname{Aut}_{F}(A)$ has a cyclic subgroup of automorphisms of order $m$ which are all extended from $i d_{D}$, and if $A$ is a free left $D$-module of rank $m$ [5]. For instance, if $F$ contains a primitive $m$ th root of unity, then generalized cyclic algebras $(D, \sigma, a)$ are cyclic extensions of $D$ of degree $m$ [5, Theorem 6]. We recall that a generalized cyclic algebra $(D, \sigma, a)$ is a quotient algebra $D[t ; \sigma] /\left(t^{m}-a\right) D[t ; \sigma]$, where $D[t ; \sigma]$ is a twisted polynomial ring, $\sigma \in \operatorname{Aut}(D)$ is an automorphism such that $\left.\sigma\right|_{K}$ has finite order $m, F_{0}=\operatorname{Fix}(\sigma) \cap K$, and $f(t)=t^{m}-a \in D[t ; \sigma]$ with $d \in F_{0}^{\times}$. The special case where $D=F$ and $F_{0}=\operatorname{Fix}(\sigma)$ yields the cyclic algebra $\left(F / F_{0}, \sigma, a\right)[12$, p. 19].

A finite-dimensional central simple algebra $A$ over $F$ is called a $G$-crossed product if it contains a maximal field extension $K / F$ which is Galois with Galois group $G=\operatorname{Gal}(K / F)$. If $G$ is solvable then $A$ is called a solvable $G$-crossed product. In [9] we revisited a result by Albert [1] on solvable crossed products and gave a proof for Albert's result using generalized cyclic algebras following Petit's approach [17], proving that a $G$-crossed product is solvable if and only if it can be constructed as a chain of generalized cyclic algebras. Hence any solvable $G$-crossed product division algebra is always a generalized cyclic division algebra. In particular, hence if $F$ contains a primitive $m$ th root of unity, solvable crossed product division algebras over $F$ are non-commutative cyclic extensions.

2010 Mathematics Subject Classification. Primary: 17A35; Secondary: 17A60, 16S36.

Key words and phrases. Skew polynomial, Ore polynomial, cyclic algebra, cyclic extension. 
A generalization of associative cyclic extensions of simple rings instead of division rings was considered in [13].

In this paper, we define and investigate nonassociative cyclic extensions of degree $m$ of both fields and central simple algebras employing nonassociative generalized cyclic division algebras: Let $A$ be a unital nonassociative division algebra. Then $A$ is called a nonassociative cyclic extension of $D$ of degree $m$, if $A$ is a free left $D$-module of rank $m$ and $\operatorname{Aut}(A)$ has a cyclic subgroup $G$ of order $m$, such that for all $H \in G,\left.H\right|_{D}=i d_{D}$.

We show that if $F$ contains a primitive $m$ th root of unity (i.e., $F$ has characteristic 0 or characteristic $p$ with $\operatorname{gcd}(m, p)=1)$, then the nonassociative generalized cyclic division algebras $(D, \sigma, a)=D[t ; \sigma] /\left(t^{m}-a\right) D[t ; \sigma]$ with $a \in D^{\times}$are nonassociative cyclic extensions of $D$ of degree $m$. Additionally, the subgroup of order $m$ in $\operatorname{Aut}_{F_{0}}(D, \sigma, a)$ that consists of automorphisms extending $i d_{D}$ contains only inner automorphisms (Corollary 10). We also investigate the structure of the automorphism groups of nonassociative generalized cyclic algebras in general.

Note that nonassociative cyclic division algebras $(K / F, \sigma, a)$ are a special case of nonassociative generalized cyclic division algebras. If $F$ contains a primitive $m$ th root of unity the nonassociative cyclic division algebras $(K / F, \sigma, a)$ are nonassociative cyclic extensions of $K$ of degree $m$. The subgroup of the automorphisms extending $i d_{K}$ has order $m$, is isomorphic to $\operatorname{ker}\left(N_{K / F}\right)$, and contains only inner automorphisms. If $F$ has no non-trivial $m$ th root of unity and $a \in K^{\times}$is not contained in any proper subfield of $K$, all automorphisms of $(K / F, \sigma, a)$ are inner and leave $i d_{K}$ fixed (Theorem 3).

We point out that nonassociative generalized cyclic algebras have been recently successfully used both in constructing space-time block codes and linear codes [19, 20, 21, 22].

The paper is organized as follows: After introducing the basic terminology in Section 1, we define nonassociative cyclic extensions and nonassociative generalized cyclic algebras in Section 2 and investigate nonassociative cyclic extensions of a field. In Section 3 we show when generalized cyclic division algebras $(D, \sigma, d)$ are nonassociative cyclic extensions of $D$ of degree $m$. We briefly look at the question when a nonassociative overring is a nonassociative cyclic extension of a field or a central simple algebra in Section 4.

The results presented in this paper complements the ones for the nonassociative algebras $(K, \delta, d)=K[t ; \delta] / K[t ; \delta] f(t)$ for $f(t)=t^{p}-t-d \in K[t ; \delta]$ constructed using a field $K$ of characteristic $p$ together with some derivation $\delta$ with minimum polynomial $g(t)=t^{p}-t \in$ $F[t], F=\operatorname{Const}(\delta)$, and of the nonassociative algebras $(D, \delta, d)=D[t ; \delta] / D[t ; \delta] f(t)$ for $f(t)=t^{p}-t-d \in D[t ; \delta]$ constructed using a division algebra $D$, where the derivation $\delta$ has minimum polynomial $g(t)=t^{p}-t \in F[t]$ and the field $F$ characteristic $p$. [18].

\section{Preliminaries}

1.1. Nonassociative algebras. Let $F$ be a field and let $A$ be an $F$-vector space. $A$ is an algebra over $F$ if there exists an $F$-bilinear map $A \times A \rightarrow A,(x, y) \mapsto x \cdot y$, denoted simply by juxtaposition $x y$, the multiplication of $A$. An algebra $A$ is called unital if there is an 
element in $A$, denoted by 1 , such that $1 x=x 1=x$ for all $x \in A$. We will only consider unital algebras without saying so explicitly.

The associator of $A$ is given by $[x, y, z]=(x y) z-x(y z)$. The left nucleus of $A$ is defined as $\operatorname{Nuc}_{l}(A)=\{x \in A \mid[x, A, A]=0\}$, the middle nucleus of $A$ is $\operatorname{Nuc}_{m}(A)=\{x \in A \mid[A, x, A]=$ $0\}$ and the right nucleus of $A$ is $\operatorname{Nuc}_{r}(A)=\{x \in A \mid[A, A, x]=0\} . \operatorname{Nuc}_{l}(A), \operatorname{Nuc}_{m}(A)$, and $\operatorname{Nuc}_{r}(A)$ are associative subalgebras of $A$. Their intersection $\operatorname{Nuc}(A)=\{x \in A \mid[x, A, A]=$ $[A, x, A]=[A, A, x]=0\}$ is the nucleus of $A$. $\operatorname{Nuc}(A)$ is an associative subalgebra of $A$ containing $F 1$ and $x(y z)=(x y) z$ whenever one of the elements $x, y, z$ lies in $\operatorname{Nuc}(A)$. The center of $A$ is $\mathrm{C}(A)=\{x \in A \mid x \in \operatorname{Nuc}(A)$ and $x y=y x$ for all $y \in A\}$.

An algebra $A \neq 0$ is called a division algebra if for any $a \in A, a \neq 0$, the left multiplication with $a, L_{a}(x)=a x$, and the right multiplication with $a, R_{a}(x)=x a$, are bijective. If $A$ has finite dimension over $F, A$ is a division algebra if and only if $A$ has no zero divisors [24, pp. $15,16]$. An element $0 \neq a \in A$ has a left inverse $a_{l} \in A$, if $R_{a}\left(a_{l}\right)=a_{l} a=1$, and a right inverse $a_{r} \in A$, if $L_{a}\left(a_{r}\right)=a a_{r}=1$. If $m_{r}=m_{l}$ then we denote this element by $m^{-1}$.

An automorphism $G \in \operatorname{Aut}_{F}(A)$ is an inner automorphism if there is an element $m \in A$ with left inverse $m_{l}$ such that $G(x)=\left(m_{l} x\right) m$ for all $x \in A$. We denote such an automorphism by $G_{m}$. The set of inner automorphisms $\left\{G_{m} \mid m \in \operatorname{Nuc}(A)\right.$ invertible $\}$ is a subgroup of $\operatorname{Aut}_{F}(A)$. Note that if the nucleus of $A$ is commutative, then for all $0 \neq n \in \operatorname{Nuc}(A)$, $G_{n}(x)=\left(n^{-1} x\right) n=n^{-1} x n$ is an inner automorphism of $A$ such that $\left.G_{n}\right|_{\operatorname{Nuc}(A)}=i d_{\operatorname{Nuc}(A)}$.

1.2. Division algebras obtained from twisted polynomial rings. Let $D$ be a unital division ring and $\sigma$ a ring automorphism of $D$. The twisted polynomial ring $D[t ; \sigma]$ is the set of polynomials $a_{0}+a_{1} t+\cdots+a_{n} t^{n}$ with $a_{i} \in D$, where addition is defined term-wise and multiplication by $t a=\sigma(a) t \quad(a \in D)$ [16]. That means, $a t^{n} b t^{m}=a \sigma^{n}(b) t^{n+m}$ and $t^{n} a=$ $\sigma^{n}(a) t^{n}$ for all $a, b \in D[12$, p. 2]. $R=D[t ; \sigma]$ is a left principal ideal domain and there is a right division algorithm in $R$, i.e. for all $g, f \in R, f \neq 0$, there exist unique $r, q \in R$ such that $\operatorname{deg}(r)<\operatorname{deg}(f)$ and $g=q f+r$ [12, p. 3]. (Our terminology is the one used by Petit [17] and different from Jacobson's [12], who calls what we call right a left division algorithm and vice versa.)

An element $f \in R$ is irreducible in $R$ if it is no unit and it has no proper factors, i.e there do not exist $g, h \in R$ such that $f=g h[12$, p. 11].

Let $f \in D[t ; \sigma]$ be of degree $m$ and let $\bmod _{r} f$ denote the remainder of right division by $f$. Then the vector space $R_{m}=\{g \in D[t ; \sigma] \mid \operatorname{deg}(g)<m\}$ together with the multiplication

$$
g \circ h=g h \bmod _{r} f
$$

becomes a unital nonassociative algebra $S_{f}=\left(R_{m}, \circ\right)$ over $F_{0}=\{z \in D \mid z h=h z$ for all $h \in$ $\left.S_{f}\right\}$ (cf. [17, (7)]), and $F_{0}$ is a subfield of $D$. We also denote this algebra $R / R f$.

We note that when $\operatorname{deg}(g)+\operatorname{deg}(h)<m$, the multiplication of $g$ and $h$ in $S_{f}$ is the same as the multiplication of $g$ and $h$ in $R$ [17, (10)].

A twisted polynomial $f \in R$ is right-invariant if $f R \subset R f$. If $f$ is right invariant then $R f$ is a two-sided ideal and conversely, every two-sided ideal in $R$ arises this way.

$S_{f}$ is associative if and only if $f$ is right-invariant. In that case, $S_{f}$ is the usual quotient algebra $D[t ; \delta] /(f)[17,(9)]$. 


\section{NonASSOCIATIVE GENERALIZED CYCLIC ALGEBRAS AND NONASSOCIATIVE CYCLIC EXTENSIONS}

In the following, let $D$ be a division algebra which is finite-dimensional over its center $F=\mathrm{C}(D)$ and $\sigma \in \operatorname{Aut}(D)$ such that $\left.\sigma\right|_{F}$ has finite order $m$ and fixed field $F_{0}=\operatorname{Fix}(\sigma) \cap F$. Note that $F / F_{0}$ is automatically a cyclic Galois field extension of degree $m$ with $\operatorname{Gal}\left(F / F_{0}\right)=$ $\left\langle\left.\sigma\right|_{F}\right\rangle$.

2.1. Following Jacobson [12, p. 19], an (associative) generalized cyclic algebra is an associative algebra $S_{f}=D[t ; \sigma] / D[t ; \sigma] f$ constructed using a right-invariant twisted polynomial

$$
f(t)=t^{m}-d \in D[t ; \sigma]
$$

with $d \in F_{0}^{\times}$. We write $(D, \sigma, d)$ for this algebra. If $D$ is a central simple algebra over $F$ of degree $n$, then $(D, \sigma, d)$ is a central simple algebra over $F_{0}$ of degree $m n$ and the centralizer of $D$ in $(D, \sigma, d)$ is $F\left[12\right.$, p. 20]. In particular, if $D=F, F / F_{0}$ is a cyclic Galois extension of degree $m$ with Galois group generated by $\sigma$ and $f(t)=t^{m}-d \in F[t ; \sigma]$, we obtain the cyclic algebra $\left(F / F_{0}, \sigma, d\right)$ of degree $m$.

This definition generalizes to nonassociative algebras as follows:

Definition 1. A nonassociative generalized cyclic algebra of degree $m n$ is an algebra $S_{f}=$ $D[t ; \sigma] / D[t ; \sigma] f$ over $F_{0}$ with $f(t)=t^{m}-d \in D[t ; \sigma], d \in D^{\times}$. We denote this algebra by $(D, \sigma, d)$.

The algebra $A=(D, \sigma, d), d \in D^{\times}$, has dimension $m^{2} n^{2}$ over $F_{0}$. In particular, if $D=F$ and $F / F_{0}$ is a cyclic Galois extension of degree $m$ with Galois group generated by $\sigma$, then $\left(F / F_{0}, \sigma, d\right)$ is a nonassociative cyclic algebra [25]. $A$ is associative if and only if $d \in F_{0}$. If $(D, \sigma, d)$ is not associative then $\operatorname{Nuc}_{l}(A)=\operatorname{Nuc}_{m}(A)=D$ and $\operatorname{Nuc}_{r}(A)=\left\{g \in S_{f} \mid f g \in\right.$ $R f\}$.

$(D, \sigma, d)$ is a division algebra over $F_{0}$ if and only if $f(t)=t^{m}-d \in D[t ; \sigma]$ is irreducible $[17,(7)]$. Moreover, we know that $f(t)=t^{2}-d \in D[t ; \sigma]$ is irreducible if and only if $\sigma(z) z \neq d$ for all $z \in D, f(t)=t^{3}-d \in D[t ; \sigma]$ is irreducible if and only if $d \neq \sigma^{2}(z) \sigma(z) z$ for all $z \in D$, and $f(t)=t^{4}-d \in D[t ; \sigma]$ is irreducible if and only if

$$
\sigma^{2}(y) \sigma(y) y+\sigma^{2}(x) y+\sigma^{2}(y) \sigma(x) \neq 0 \text { or } \sigma^{2}(x) x+\sigma^{2}(y) \sigma(y) x \neq d
$$

for all $x, y \in D$ (cf. [17] or [19], [7, Theorem 3.19], see also [11]). More generally, if $F_{0}$ contains a primitive $m$ th root of unity and $m$ is prime then $f(t)=t^{m}-d \in D[t ; \sigma]$ is irreducible if and only if $d \neq \sigma^{m-1}(z) \cdots \sigma(z) z$ for all $z \in D$ ([7, Theorem 3.11], see also [19, Theorem 6]).

Amitsur's definition [5] of cyclic extensions generalizes to the nonassociative setting as follows:

Definition 2. Let $m \geq 2$. Let $A$ be a nonassociative division algebra with center $F_{0}$ and $D$ an associative division algebra with center $F$. Then $A$ is a nonassociative cyclic extension of $D$ of degree $m$, if $A$ is a free left $D$-module of rank $m$ and $\operatorname{Aut}(A)$ has a cyclic subgroup $G$ of order $m$, such that for all $H \in G,\left.H\right|_{D}=i d$. 
2.2. Nonassociative cyclic extensions of a field. For a nonassociative cyclic algebra $(K / F, \sigma, d)$ of degree $m$, and for all $k \in K$ such that $N_{K / F}(k)=1$, the map

$$
H_{i d, k}\left(\sum_{i=0}^{m-1} a_{i} t^{i}\right)=a_{0}+\sum_{i=1}^{m-1} a_{i}\left(\prod_{l=0}^{i-1} \sigma^{l}(k)\right) t^{i}
$$

is an inner $F$-automorphism of $(K / F, \sigma, d)$ extending $i d_{K}$. The subgroup generated by the automorphisms $H_{i d, k}$ is isomorphic to $\operatorname{ker}\left(N_{K / F}\right)$ [8, Theorem 19].

The maps $H_{i d, k}$ are the only $F$-automorphisms of $(K / F, \sigma, d)$, unless for some $j \in$ $\{1, \ldots, m-1\}, \sigma^{j}$ can be extended to an $F$-automorphism of $(K / F, \sigma, d)$ as well. More precisely, the automorphism $\tau=\sigma^{j}$ with $j \in\{1, \ldots, m-1\}$ can be extended to an $F$ automorphism $H$ of $(K / F, \sigma, d)$, if and only if there is an element $k \in K$ such that

$$
\sigma^{j}(d)=N_{K / F}(k) d
$$

The extension then has the form $H=H_{\tau, k}$ with

$$
H_{\tau, k}\left(\sum_{i=0}^{m-1} a_{i} t^{i}\right)=\tau\left(a_{0}\right)+\sum_{i=1}^{m-1} \tau\left(a_{i}\right)\left(\prod_{l=0}^{i-1} \sigma^{l}(k)\right) t^{i}
$$

$[8$, Theorem 4]. We then immediately get the following partial generalization of [5, Theorem $6]$ :

Theorem 1. Suppose $F$ contains a primitive mth root of unity $\omega, A=(K / F, \sigma, d)$ is a nonassociative cyclic division algebra of degree $m$ over $F$, and $d \in K \backslash F$. Then $A$ is a nonassociative cyclic extension of $K$ of degree $m$. The generating automorphism of the subgroup of $\operatorname{Aut}_{F}(A)$ of order $m$ is given by $H_{i d, \omega}$.

Proof. $\left\langle H_{\mathrm{id}, \omega}\right\rangle$ is a cyclic subgroup of $\operatorname{Aut}_{F}(A)$ of order $m$ by [8, Theorem 20]. It consists of automorphisms extending $i d_{K}$, therefore $A$ is a nonassociative cyclic extension of $K$.

Corollary 2. If $m$ is prime, $F$ contains a primitive $m$ th root of unity and $K / F$ is a cyclic Galois extension of degree $m$, then $K$ has a nonassociative cyclic extension of degree $m$.

Proof. Let $d \in K \backslash F$ and suppose $\operatorname{Gal}(K / F)=\langle\sigma\rangle$. Then since $m$ is prime, the nonassociative cyclic algebra $A=(K / F, \sigma, d)$ is a division algebra [25, Corollary 4.5]. Thus $A$ is a nonassociative cyclic extension of $K$ by Theorem 1 .

If $F$ has no non-trivial $m$ th root of unity, we obtain:

Theorem 3. Suppose $F$ has no non-trivial mth root of unity. Let $A=(K / F, \sigma, d)$ be a nonassociative cyclic algebra of degree $m$ where $d \in K^{\times}$is not contained in any proper subfield of $K$. Then every $F$-automorphism of $A$ leaves $K$ fixed and

$$
\operatorname{Aut}_{F}(A) \cong \operatorname{ker}\left(N_{K / F}\right) \text {. }
$$

In particular, all automorphisms of $A$ are inner. 
Proof. Every automorphism of $A$ has the form $H_{i d, k}$ : suppose that there exist $j \in\{1, \ldots, m-$ $1\}$ and $k \in K^{\times}$such that $H_{\sigma^{j}, k} \in \operatorname{Aut}_{F}(A)$. This implies $H_{\sigma^{j}, k}^{2}=H_{\sigma^{j}, k} \circ H_{\sigma^{j}, k} \in \operatorname{Aut}_{F}(A)$ and

$$
H_{\sigma^{j}, k}^{2}\left(\sum_{i=0}^{m-1} x_{i} t^{i}\right)=\sigma^{2 j}\left(x_{0}\right)+\sum_{i=1}^{m-1} \sigma^{2 j}\left(x_{i}\right)\left(\prod_{q=0}^{i-1} \sigma^{j+q}(k) \sigma^{q}(k)\right) t^{i}
$$

Now $H_{\sigma^{j}, k}^{2}$ must have the form $H_{\sigma^{2 j}, l}$ for some $l \in K^{\times}$, and comparing (2) and (3) yields $l=k \sigma^{j}(k)$. Similarly, $H_{\sigma^{j}, k}^{3}=H_{\sigma^{3 j}, s} \in \operatorname{Aut}_{F}(A)$ where $s=k \sigma^{j}(k) \sigma^{2 j}(k)$. Continuing in this manner we conclude that the automorphisms $H_{\sigma^{j}, k}, H_{\sigma^{2 j}, l}, H_{\sigma^{3 j}, s}, \ldots$ all satisfy (1) implying that

$$
\begin{aligned}
\sigma^{j}(d) & =N_{K / F}(k) d, \\
\sigma^{2 j}(d) & =N_{K / F}\left(k \sigma^{j}(k)\right) d=N_{K / F}(k)^{2} d, \\
\vdots & \vdots \\
d=\sigma^{n j}(d) & =N_{K / F}(k)^{n} d,
\end{aligned}
$$

where $n=m / \operatorname{gcd}(j, m)$ is the order of $\sigma^{j}$. Note that $\sigma^{i j}(d) \neq d$ for all $i \in\{1, \ldots, n-1\}$ since $d$ is not contained in any proper subfield of $K$. Therefore $N_{K / F}(k)^{n}=1$ and $N_{K / F}(k)^{i} \neq 1$ for all $i \in\{1, \ldots, n-1\}$ by (4), i.e. $N_{K / F}(k)$ is a primitive $n$th root of unity, thus also an $m$ th root of unity, a contradiction. This proves the assertion.

Note that if $d \in K^{\times}$is not contained in any proper subfield of $K$ then $1, d, \ldots, d^{m-1}$ are linearly independent over $F$ and thus $A$ is a division algebra [25]. In particular, if $m$ is prime then $1, d, \ldots, d^{m-1}$ are linearly independent over $F$. This yields for a field $F$ of arbitrary characteristic:

Corollary 4. Suppose that $F$ has no non-trivial mth root of unity. If $d \in K^{\times}$is not contained in any proper subfield of $K$ (e.g. if $m$ is prime), and $\operatorname{ker}\left(N_{K / F}\right)$ has a subgroup of order $m$, then any cyclic algebra $A=(K / F, \sigma, d)$ is a cyclic extension of $K$ of degree $m$.

Example 5. Let $K=\mathbb{F}_{q^{m}}$ be a finite field, $q=p^{r}$ for some prime $p, \sigma$ an automorphism of $K$ of order $m \geq 2$ and $F=\operatorname{Fix}(\sigma)=\mathbb{F}_{q}$, i.e. $K / F$ is a cyclic Galois extension of degree $m$ with $\operatorname{Gal}(K / F)=\langle\sigma\rangle$. Then $\operatorname{ker}\left(N_{K / F}\right)$ is a cyclic group of order $s=\left(q^{m}-1\right) /(q-1)$ and any division algebra $(K / F, \sigma, d)$ has exactly $s$ inner automorphisms, all of them extending $i d_{K}$. The subgroup they generate is cyclic and isomorphic to $\operatorname{ker}\left(N_{K / F}\right)$ [10]. Hence if $m$ divides $s$, which is the case if $F$ contains a primitive $m$ th root of unity, then there is a subgroup of automorphisms of order $m$ extending $i d_{K}$ and hence $(K / F, \sigma, d)$ is a cyclic extension of $K$ of degree $m$.

\section{Nonassociative CyClic extensions of a Central Simple Algebra}

3.1. From now until stated otherwise, let $A=(D, \sigma, d)$ be a nonassociative generalized cyclic algebra of degree $m n$ over $F_{0}$, for some $d \in D \backslash F_{0}$. We first determine the automorphisms of $A$ : 
Theorem 6. (i) Suppose $\tau \in \operatorname{Aut}_{F_{0}}(D)$ commutes with $\sigma$. Then $\tau$ can be extended to an automorphism $H \in \operatorname{Aut}_{F_{0}}(A)$, if and only if there is some $k \in F^{\times}$such that $\tau(d)=$ $N_{F / F_{0}}(k) d$. In that case, the extension $H$ of $\tau$ has the form $H=H_{\tau, k}$ with

$$
H_{\tau, k}\left(\sum_{i=0}^{m-1} a_{i} t^{i}\right)=\tau\left(a_{0}\right)+\sum_{i=1}^{m-1} \tau\left(a_{i}\right)\left(\prod_{l=0}^{i-1} \sigma^{l}(k)\right) t^{i} .
$$

All maps $H_{\tau, k}$ where $\tau \in \operatorname{Aut}_{F_{0}}(D)$ commutes with $\sigma$ and where $k \in F^{\times}$such that $\tau(d)=$ $N_{F / F_{0}}(k) d$ (hence $N_{F / F_{0}}(k)^{m n}=1$ ), are automorphisms of $A$.

In particular, for $\tau \neq i d$ and $d \notin \operatorname{Fix}(\tau), N_{F / F_{0}}(k) \neq 1$.

(ii) $i d \in \operatorname{Aut}(D)$ can be extended to an automorphism $H \in \operatorname{Aut}_{F_{0}}(A)$, if and only if there is some $k \in F^{\times}$such that $N_{F / F_{0}}(k)=1$. In that case, the extension $H$ of id has the form $H=H_{i d, k}$ with

$$
H_{i d, k}\left(\sum_{i=0}^{m-1} a_{i} t^{i}\right)=a_{0}+\sum_{i=1}^{m-1} a_{i}\left(\prod_{l=0}^{i-1} \sigma^{l}(k)\right) t^{i} .
$$

All $H_{i d, k}$ where $k \in F^{\times}$such that $N_{F / F_{0}}(k)=1$ are automorphisms of $A$.

Proof. (i) Let $H \in \operatorname{Aut}_{F_{0}}(A)$, then $\left.H\right|_{D} \in \operatorname{Aut}_{F_{0}}(D)$, since $H$ leaves the left nucleus invariant. Thus $\left.H\right|_{D}=\tau$ for some $\tau \in \operatorname{Aut}_{F_{0}}(D)$. Write $H(t)=\sum_{i=0}^{m-1} k_{i} t^{i}$ for some $k_{i} \in D$, then we have

$$
H(t z)=H(t) H(z)=\left(\sum_{i=0}^{m-1} k_{i} t^{i}\right) \tau(z)=\sum_{i=0}^{m-1} k_{i} \sigma^{i}(\tau(z)) t^{i}
$$

and

$$
H(t z)=H(\sigma(z) t)=\tau(\sigma(z)) \sum_{i=0}^{m-1} k_{i} t^{i}=\sum_{i=0}^{m-1} \tau(\sigma(z)) k_{i} t^{i}
$$

for all $z \in D$. Comparing the coefficients of $t^{i}$ yields

$$
k_{i} \sigma^{i}(\tau(z))=k_{i} \tau\left(\sigma^{i}(z)\right)=\tau(\sigma(z)) k_{i} \text { for all } i=\{0, \ldots, m-1\}
$$

for all $z \in D$ since $\sigma$ and $\tau$ commute. In particular, we obtain

$$
k_{i}\left(\tau\left(\sigma^{i}(z)\right)-\tau(\sigma(z))\right)=0 \text { for all } i \in\{0, \ldots, m-1\}
$$

for all $z \in F$, i.e. $k_{i}=0$ or $\left.\sigma\right|_{F}=\left.\sigma^{i}\right|_{F}$ for all $i \in\{0, \ldots, m-1\}$. As $\left.\sigma\right|_{F}$ has order $m$, this means $k_{i}=0$ for all $1 \neq i \in\{0, \ldots, m-1\}$. For $i=1$, this yields $k_{1} \tau(\sigma(z))=\tau(\sigma(z)) k_{1}$ for all $z \in D$, hence $k_{1} \in F$. This implies $H(t)=k t$ for some $k \in F^{\times}$.

Since

$$
H\left(z t^{i}\right)=H(z) H(t)^{i}=\tau(z)(k t)^{i}=\tau(z)\left(\prod_{l=0}^{i-1} \sigma^{l}(k)\right) t^{i},
$$

for all $i \in\{1, \ldots, m-1\}$ and all $z \in D, H$ has the form

$$
H_{\tau, k}: \sum_{i=0}^{m-1} a_{i} t^{i} \mapsto \tau\left(a_{0}\right)+\sum_{i=1}^{m-1} \tau\left(a_{i}\right)\left(\prod_{l=0}^{i-1} \sigma^{l}(k)\right) t^{i},
$$

for some $k \in F^{\times}$.

Comparing the constant terms in $H(t)^{m}=H\left(t^{m}\right)=H(d)$ implies

$$
\tau(d)=k \sigma(k) \cdots \sigma^{m-1}(k) d=N_{F / F_{0}}(k) d .
$$


Let $N=N_{F / F_{0}} \circ N_{D / F}$ be the norm of the $F_{0}$-algebra $D$. Applying $N$ to both sides of the equation yields $N(d)=N(k)^{m} N(d)$, so that $N(k)^{m}=1$. Now $k \in F^{\times}$and $D$ has degree $n$, thus

$$
N(k)=N_{F / F_{0}}\left(N_{D / F}(k)\right)=N_{F / F_{0}}\left(k^{n}\right)=N_{F / F_{0}}(k)^{n},
$$

and so $N(k)^{m}=N_{F / F_{0}}(k)^{n m}=1$.

Finally, the fact that the maps $H_{\tau, k}$ are automorphisms when $\tau$ commutes with $\sigma$, and $\tau(d)=N_{F / F_{0}}(k) d$, can be shown similarly to the proof of [8, Theorem 4], see also [7].

(ii) In particular, for $\tau=i d$, we get from (i) that $H$ has the form

$$
H_{i d, k}: \sum_{i=0}^{m-1} a_{i} t^{i} \mapsto a_{0}+\sum_{i=1}^{m-1} a_{i}\left(\prod_{l=0}^{i-1} \sigma^{l}(k)\right) t^{i}
$$

for some $k \in F^{\times}$with $k \sigma(k) \cdots \sigma^{m-1}(k)=N_{F / F_{0}}(k)=1$.

The above is proved for a more general set-up in the first author's $\mathrm{PhD}$ thesis [7]. Note that the automorphisms $H_{\tau, k}$ are restrictions of automorphisms of the twisted polynomial ring $D[t ; \sigma]$.

Corollary 7. (i) The subgroup of $F_{0}$-automorphisms of $A$ extending $i d_{D} \in \operatorname{Aut}_{F_{0}}(D)$ is isomorphic to

$$
\left\{k \in F^{\times} \mid k \sigma(k) \cdots \sigma^{m-1}(k)=1\right\} .
$$

(ii) If $F_{0}$ contains a primitive mth root of unity $\omega$, then $\left\langle H_{i d, \omega}\right\rangle$ is a cyclic subgroup of $\operatorname{Aut}_{F_{0}}(A)$ of order $m$.

3.2. We obtain the following generalization of [5, Theorem 6$]$ :

Corollary 8. Suppose $F_{0}$ contains a primitive mth root of unity. If $f(t)=t^{m}-d \in D[t ; \sigma]$ is irreducible, then $A$ is a nonassociative cyclic extension of $D$ of degree $m$. In particular, if $m$ is prime and

$$
d \neq \sigma^{m-1}(z) \cdots \sigma(z) z
$$

for all $z \in D$, then $A$ is a nonassociative cyclic extension of $D$ of degree $m$.

Proof. If $F_{0}$ contains a primitive $m$ th root of unity $\omega$, then $\left\langle H_{i d, \omega}\right\rangle$ is a cyclic subgroup of $\operatorname{Aut}_{F_{0}}(A)$ of order $m$ by Corollary 7 (ii). If $m$ is prime, then $f(t)=t^{m}-d \in D[t ; \sigma]$ is irreducible if and only if

$$
d \neq \sigma^{m-1}(z) \cdots \sigma(z) z
$$

for all $z \in D$. The rest is trivial.

Proposition 9. Every automorphism $H_{i d, k}$ of $A$ is an inner automorphism

$$
G_{c}\left(\sum_{i=0}^{m-1} a_{i} t^{i}\right)=\left(c^{-1} \sum_{i=0}^{m-1} a_{i} t^{i}\right) c
$$

for some $c \in F^{\times}$satisfying $k=\sigma(c) c^{-1}$. 
Proof. For all $k \in F$ such that $N_{F / F_{0}}(k)=1, H_{i d, k}$ is an $F$-automorphism extending $i d_{D}$. These are the only $F_{0}$-automorphisms of $A$, unless $\tau \neq i d$ can be also extended. By Hilbert's Satz $90, N_{F / F_{0}}(k)=1$ if and only if there is $c \in F^{\times}$such that $k=c^{-1} \sigma(c)$ [14]. So there is $c \in F^{\times}$such that $k=c^{-1} \sigma(c)$ and

$$
k \sigma(k) \cdots \sigma^{i-1}(k)=c \sigma^{i}(c), \quad i=1 \ldots, m-1
$$

yields that $H_{i d, k}=G$ with

$$
G\left(\sum_{i=0}^{m-1} a_{i} t^{i}\right)=a_{0}+a_{1} c^{-1} \sigma(c) t+\sum_{i=2}^{m-1} a_{i} c^{-1} \sigma^{i}(c) t^{i},
$$

which is an inner automorphism, since $G=G_{c}$ with

$$
G_{c}\left(\sum_{i=0}^{m-1} a_{i} t^{i}\right)=\left(c^{-1} \sum_{i=0}^{m-1} a_{i} t^{i}\right) c .
$$

Note that here we use that $F=C(D)$.

Corollary 10. If $F_{0}$ contains a primitive mth root of unity $\omega$, then $A$ is a cyclic extension of $D$ of order $m$, and all automorphisms extending $i d_{D}$ are inner.

Example 11. Let $F$ and $L$ be fields and let $K$ be a cyclic Galois extension of both $F$ and $L$ such that $[K: F]=n,[K: L]=m, \operatorname{Gal}(K / F)=\langle\gamma\rangle$ and $\operatorname{Gal}(K / L)=\langle\sigma\rangle$, and $\sigma \circ \gamma=\gamma \circ \sigma$. Define $F_{0}=F \cap L$.

Let $D=(K / F, \gamma, c)$ be a cyclic division algebra of degree $n$ with $c \in F_{0}$, i.e. $D \cong D_{0} \otimes_{F_{0}} K$ for some cyclic algebra $D_{0}=\left(F / F_{0}, \gamma, c\right)$. Let $1, e \ldots, e^{n-1}$ be the canonical basis of $D$, that is $e^{n}=c, e x=\gamma(x) e$ for every $x \in K$. For $x=x_{0}+x_{1} e+x_{2} e^{2}+\cdots+x_{n-1} e^{n-1} \in D$, define an $L$-linear map $\sigma \in \operatorname{Aut}_{L}(D)$ via

$$
\sigma(x)=\sigma\left(x_{0}\right)+\sigma\left(x_{1}\right) e+\sigma\left(x_{2}\right) e^{2}+\cdots+\sigma\left(x_{n-1}\right) e^{n-1}
$$

(note that $c \in L$ implies $\sigma(x y)=\sigma(x) \sigma(y)$ for all $x, y \in D$ ). Then $\sigma \in \operatorname{Aut}_{F_{0}}(D)$ has order $m$. For all $d \in D^{\times}$,

$$
D[t ; \sigma] / D[t ; \sigma]\left(t^{m}-d\right)=(D, \sigma, d)
$$

is a generalized nonassociative cyclic algebra of degree $m n$ over $F_{0}$ (used for instance in $[20]) .(D, \sigma, d)$ is associative if and only if $d \in F_{0}$. In the special case that $d \in F^{\times}$,

$$
(D, \sigma, d)=\left(L / F_{0}, \gamma, c\right) \otimes_{F_{0}}\left(F / F_{0}, \sigma, d\right)
$$

is the tensor product of an associative and a nonassociative cyclic algebra.

If $F_{0}$ contains a primitive $m$ th root of unity and $d \in D^{\times} \backslash F_{0}$ is chosen such that $f(t)=$ $t^{m}-d \in D[t ; \sigma]$ is irreducible, then $(D, \sigma, d)$ is a cyclic extension of $D$ of order $m$, and all automorphisms extending $i d_{D}$ are inner (Corollary 10). Recall that if $m$ is prime then $f(t)=t^{m}-d \in D[t ; \sigma]$ is irreducible if and only if $d \neq \sigma^{m-1}(z) \cdots \sigma(z) z$ for all $z \in D$.

For $m=2$, this algebra is studied in [21], and used in the codes constructed in [15]. For $d \in F^{\times}$the algebra is used in [23], see also [20]. 
3.3. In the following, let $D$ be a division algebra which is finite-dimensional over its center $F=C(D), \sigma \in \operatorname{Aut}(D)$ an automorphism such that $\left.\sigma\right|_{F}$ has finite order $q$ and fixed field $F_{0}=\operatorname{Fix}(\sigma) \cap F$. If $D$ has degree $n$ then the associative generalized cyclic algebra $A=(D, \sigma, a)$ has degree $q n$ over $F_{0}$. We choose $a \in F_{0}$ such that $A$ is a division algebra.

Now assume $F_{0}$ contains a primitive $q$ th root of unity $\omega$. Then $\tau=H_{i d_{D}, \omega}: A \rightarrow A$ generates a cyclic subgroup of $\operatorname{Aut}_{F_{0}}(A)$ of order $q$ by [5, Theorem 6] which consists of automorphisms which all extend $i d_{D}$. We obtain the following generalization of $[5$, Theorem 7]:

Theorem 12. Suppose there exists $\rho \in \operatorname{Aut}(A), b \in A$ and $1 \neq k \in F_{0}$ such that

(1) $\tau$ commutes with $\rho$,

(2) $\tau(b)=k \rho(k) \cdots \rho^{m-1}(k) b$,

(3) $k^{q}$ is a primitive $m$ th root of unity,

(4) $t^{m}-b \in A[t ; \rho]$ is irreducible, and

(5) the algebra $B=A[t ; \rho] / A[t ; \rho]\left(t^{m}-b\right)$ is either associative, or finite-dimensional over $F_{0} \cap \operatorname{Fix}(\rho)$, or finite-dimensional over $\operatorname{Nuc}_{r}(B)$.

Then $B$ is a nonassociative cyclic extension of $D$ of degree $m q$ which contains $A$.

Proof. Since $B$ is a free left $A$-module of rank $m$ and $A$ is a free left $D$-module of rank $q$, $B$ is a free left $D$-module of rank $m q$. Furthermore, (4) and (5) yield that $B$ is a division algebra by $[17,(7)]$. Define the map

$$
H_{\tau, k}: B \rightarrow B, \sum_{i=0}^{m-1} x_{i} t^{i} \mapsto \tau\left(x_{0}\right)+\sum_{i=1}^{m-1} \tau\left(x_{i}\right)\left(\prod_{l=0}^{i-1} \rho^{l}(k)\right) t^{i} \quad\left(x_{i} \in A\right),
$$

then (1) and (2) together imply that $H_{\tau, k}$ is an automorphism of $B$ by [8, Theorem 4].

$H_{\tau, k}$ has order $m q$ : We have $\tau(k)=k$ because $k \in F_{0} \subset D$. Therefore straightforward calculations yield $H_{\tau, k}^{2}=H_{\tau, k} \circ H_{\tau, k}=H_{\tau^{2}, k \tau(k)}=H_{\tau^{2}, k^{2}}, H_{\tau, k}^{3}=H_{\tau^{3}, k^{3}}$ etc., thus $H_{\tau, k}$ will have order at least $q$. After $q$ steps we obtain $H_{\tau, k}^{q}=H_{i d_{A}, v}$ with $v=k^{q}$ and so $H_{\tau, k}$ has order $m q$ by (3).

Finally $\left.H_{\tau, k}\right|_{D}=\left.\tau\right|_{D}=i d_{D}$, hence we conclude $B$ is a nonassociative cyclic extension of $D$ of degree $m q$.

\section{When is A Ring a nONASSOCiative CyClic EXtension?}

A nonassociative ring $A \neq 0$ is called a right division ring, if for all $a \in A, a \neq 0$, the right multiplication with $a, R_{a}(x)=x a$, is bijective. If $D$ is a division ring and $f$ is irreducible, then $S_{f}=D[t ; \sigma] / D[t ; \sigma] f$ is a right division algebra and has no zero divisors ([17, (6)] or [11]).

Theorem 13. (cf. [17, (3), (6)])

(i) Let $S$ be a nonassociative ring with multiplication $\circ$. Suppose that

(1) $S$ has an associative subring $D$ which is a division algebra and $S$ is a free left $D$-module of rank $m$, and there is $t \in S$ such that $t^{j}, 0 \leq i<m$ is a basis of $S$ over $D$, when defining $t^{j+1}=t \circ t^{j}, t^{0}=1$;

(2) for all $a \in D, a \neq 0$, there are $a_{1}, a_{2} \in D, a_{1} \neq 0$, such that $t \circ a=a_{1} \circ t+a_{2}$; 
(3) for all $a, b, c \in D, i+j<m, k<m$, we have $\left[a \circ t^{i}, b \circ t^{j}, c \circ t^{k}\right]=0$.

Then $S \cong S_{f}$ with $f(t) \in D[t ; \sigma, \delta]$ and $\sigma, \delta$ defined via $t \circ a=\sigma(a) \circ t+\delta(a)$ and where the polynomial $f(t)=t^{m}-\sum_{i=0}^{m-1} d_{i} t^{i}$ is given by $t^{m}=\sum_{i=0}^{m-1} d_{i} t^{i}$ with $t^{0}=1, t^{i+1}=t \circ t^{i}$, $0 \leq i<m$.

(ii) If $S$ is a right division ring in (i) then $f$ is irreducible.

Theorem 13 yields the nonassociative analogues to the existence conditions for associative cyclic extensions in [5, Theorem 6].

Theorem 14. (i) Let $S$ be a nonassociative ring with multiplication $\circ$, which has a field $K$ as a subring, and is a free left $K$-vector space of dimension $m$. Suppose that

(1) there is $t \in S$ such that $t^{i}, 0 \leq i<m$, is a basis of $S$ over $K$ when defining $t^{0}=1$, $t^{i+1}=t \circ t^{i}, 0 \leq i<m$;

(2) for all $a \in K, a \neq 0$, there is $a^{\prime} \in K^{\times}$, such that $t \circ a=a^{\prime} \circ t$;

(3) for all $a, b, c \in K, i+j<m, k<m$, we have $\left[a \circ t^{i}, b \circ t^{j}, c \circ t^{k}\right]=0$;

(4) $t^{m}=d$ for some $d \in K^{\times}$;

(5) the map $\sigma: K \rightarrow K, \sigma(a)=a^{\prime}$, has order $m$ and fixed field $F=\{a \in K \mid t \circ a=a \circ t\}$ containing a primitive mth root of unity $\omega$, and $K / F$ is a finite cyclic Galois extension.

Then $S \cong S_{f}=(K / F, \sigma, d)$ with $f(t)=t^{m}-d \in K[t ; \sigma]$.

(ii) If $S$ is a right division ring in (i) then $f$ is irreducible and $S \cong(K / F, \sigma, d)$ is a nonassociative cyclic extension of $K$ of degree $m$.

Proof. (1), (2) and (3) imply that $S \cong S_{f}$ with $f \in K[t ; \sigma]$ and $\sigma$ defined via $t \circ a=\sigma(a) \circ t$, i.e. $\sigma(a)=a^{\prime}$, and where the polynomial $f(t)=t^{m}-\sum_{i=0}^{m-1} d_{i} t^{i}$ is given by $t^{m}=\sum_{i=0}^{m-1} d_{i} t^{i}$ for some suitably chosen $d_{i}$ (cf. [17, (3)]). (4) implies that indeed $f(t)=t^{m}-d$. (5) guarantees that $(K / F, \sigma, d)$ where $F$ contains a primitive $m$ th root of unity $\omega$.

(ii) Here we are in the setup of Theorem 1 which yields the assertion: $F$ contains a primitive $m$ th root of unity $\omega$, so $\left\langle H_{i d, \omega}\right\rangle$ is a cyclic subgroup of order $m$ of the division algebra $(K / F, \sigma, d)$.

For nonassociative cyclic extensions of a central simple algebra $D$ we obtain from Theorem 13:

Theorem 15. (i) Let $S$ be a nonassociative ring with multiplication $\circ$, which has an associative subring $D$ which is a division algebra and $S$ is a free left $D$-module of rank $m$. Suppose that

(1) there is $t \in S$ such that $t^{i}, 0 \leq i<m$, is a basis of $S$ over $D$ when defining $t^{0}=1$, $t^{i+1}=t \circ t^{i}, 0 \leq i<m$;

(2) for all $a \in D, a \neq 0$, there are $a^{\prime} \in D, a^{\prime} \neq 0$, such that $t \circ a=a^{\prime} \circ t$;

(3) for all $a, b, c \in D, i+j<m, k<m$, we have $\left[a \circ t^{i}, b \circ t^{j}, c \circ t^{k}\right]=0$;

(4) $t^{m}=d$;

(5) the map $\sigma: D \rightarrow D, \sigma(a)=a^{\prime}$, has order $m$, fixed field $\{a \in D \mid t \circ a=a \circ t\}$ and $D / F$ is a central simple algebra, where $F_{0}=F \cap \operatorname{Fix}(\sigma)$ contains a primitive mth root of unity $\omega$. Then $S \cong S_{f}=(D, \sigma, d)$ with $f(t)=t^{m}-d \in D[t ; \sigma]$.

(ii) If $S$ is a right division ring and $D$ a central simple algebra in (i), then $f$ is irreducible and $S$ a nonassociative cyclic extension of $D$ of degree $m$. 
Proof. (1), (2) and (3) imply that $S \cong S_{f}$ with $f \in D[t ; \sigma]$ and $\sigma$ defined via $t \circ a=\sigma(a) \circ t$, i.e. $\sigma(a)=a^{\prime}$, and where the polynomial $f(t)=t^{m}-\sum_{i=0}^{m-1} d_{i} t^{i}$ is given by $t^{m}=\sum_{i=0}^{m-1} d_{i} t^{i}$ for some suitably chosen $d_{i}$ (cf. [17, (3)]). (4) implies $f(t)=t^{m}-d$. (5) guarantees that $S \cong(D, \sigma, d)$ where $F$ contains a primitive $m$ th root of unity $\omega$.

(ii) Here we are in the setup of Theorem 6 which yields the assertion, since $F$ contains a primitive $m$ th root of unity $\omega,\left\langle H_{i d, \omega}\right\rangle$ is a cyclic subgroup of order $m$ of the division algebra $(D, \sigma, d)$.

\section{REFERENCES}

[1] A. A. Albert, Structure of algebras. Vol. 24, AMS 1939.

[2] A. A. Albert, Cyclic fields of degree $p^{n}$ over F of characteristic p. Bull. AMS 40 (1934), 625-631.

[3] A. A. Albert, Modern higher algebra. Chicago 1937, Chapter IX, 192-208.

[4] A. S. Amitsur, Differential Polynomials and Division Algebras. Annals of Mathematics, Vol. 59 (2) (1954) $245-278$

[5] A. S. Amitsur, Non-commutative cyclic fields. Duke Math. J. 21 (1954), 87105.

[6] E. Artin, O. Schreier, Über eine Kennzeichnung der reell algebraischen Körper. Abh. Math. Seminar der Hamburgischen Universitäten, 5 (1927), 225-231.

[7] C. Brown, Petit's algebras and their automorphisms. PhD Thesis, University of Nottingham, 2018.

[8] C. Brown, S. Pumplün, The automorphisms of Petit's algebras. Comm. Algebra 46 (2) (2018), 834-849.

[9] C. Brown, S. Pumplün, Solvable crossed product algebras revisited. Online at arXiv:1702.04605 [math.RA]

[10] C. Brown, S. Pumplün, A. Steele, Automorphisms and isomorphisms of Jha-Johnson semifields obtained from skew polynomial rings. To appear in Comm. Alg. Online at arXiv:1703.02356 [math.RA]

[11] C. Brown, S. Pumplün, How a nonassociative algebra reflects the properties of a skew polynomial. Preprint, 2018.

[12] N. Jacobson, "Finite-dimensional division algebras over fields." Springer Verlag, Berlin-Heidelberg-New York, 1996.

[13] K. Kishimoto, On cyclic extensions of simple rings. J. Fac. Sci. Hokkaido Univ. Ser. I 19 (1966), 74-85.

[14] T. Y. Lam, A. Leroy, Hilbert 90 theorems over division rings. Trans. Amer. Math. Soc. 345 (2) (1994), 595-622.

[15] N. Markin, F. Oggier, Iterated space-time code constructions from cyclic algebras. IEEE Transactions on Information Theory, 59 (9), September 2013.

[16] O. Ore, Theory of noncommutative polynomials. Annals of Math. 34 (3) (1933), 480-508.

[17] J.-C. Petit, Sur certains quasi-corps généralisant un type d'anneau-quotient. Séminaire Dubriel. Algèbre et théorie des nombres 20 (1966 - 67), 1-18.

[18] S. Pumplün, Nonassociative differential extensions of characteristic $p$. Results in Mathematics 72 (1-2) (2017), 245-262, DOI 10.1007/s00025-017-0656-x

[19] S. Pumplün, Tensor products of nonassociative cyclic algebras. Journal of Algebra 451 (2016), 145-165.

[20] S. Pumplün, A. Steele, Fast-decodable MIDO codes from nonassociative algebras. Int. J. of Information and Coding Theory (IJICOT) 3 (1) 2015, 15-38.

[21] S. Pumplün, How to obtain division algebras used for fast decodable space-time block codes. Adv. in Math. Comm. 8 (3) (2014), 323 - 342.

[22] S. Pumplün, A. Steele, The nonassociative algebras used to build fast-decodable space-time block codes. Adv. Math. Comm. 9 (4) (2015), 449-469.

[23] K. P. Srinath, B. S. Rajan, Fast-decodable MIDO codes with large coding gain. IEEE Transactions on Information Theory (2) 60 2014, 992-1007.

[24] R. D. Schafer, "An Introduction to Nonassociative Algebras." Dover Publ., Inc., New York, 1995.

[25] A. Steele, Nonassociative cyclic algebras. Israel Journal of Mathematics 200 (1) (2014), 361-387.

[26] E. Witt, Zyklische Körper und Algebren der Charakteristik p vom Grad p ${ }^{n}$. J. Reine Angew. Math. 176 (1936), 126-140. 
Email address: christian_jb@hotmail.co.uk; susanne.pumpluen@nottingham.ac.uk

School of Mathematical Sciences, University of Nottingham, University Park, Nottingham NG7 2RD, United Kingdom 